\title{
OPTICAL \& NEAR INFRARED IMAGING OF NGC1275
}

\author{
D. H. Hughes \& E. I. Robson \\ Physics and Astronomy, Lancashire Polytechnic, Preston PR1 2TQ, U.K. \\ M. J. Ward \\ Institute of Astronomy, Cambridge CB3 OHA, U.K.
}

\begin{abstract}
We are currently studying a selection of active galaxies using the new IR array camera IRCAM on UKIRT. Our aim is to seperate the underlying stellar emission from that of the active galactic nucleus. Although the optical is the best wavelength region to discriminate between the different populations in the underlying spiral and elliptical galaxies, it is in the infrared that the contrast between the non-thermal central core and the surrounding galaxy is increased. We present reduced data from infrared images taken at $1.25,1.65$ and $2.2 \mu \mathrm{m}$ with an image scale of $0.6 \mathrm{arcsec} / \mathrm{pixel}$ together with optical 0.44 and $0.55 \mu \mathrm{m}$ CCD images of the Seyfert galaxy NGC1275.
\end{abstract}

\section{NGC1275}

The IR images immediately reveal a similar morphology to optical CCD frames, that is a circular symmetric underlying galaxy with a bright nucleus and also a 'hotspot' 7 arcsec NE of the nucleus that has caused much discussion over the past 20 years. We shall comment in turn on the nature of all these features. The following $\mathrm{K}$-corrections and interstellar reddening corrections have been applied to all fluxes $(B-V=0.31, J-H=0.11, H-K=0.04$, Aaronson 1977). NGC1275 shows two different gas systems (Minkowski, 1957) for which one explanation is that it is an elliptical with an infalling spiral galaxy. However no Seyfert nucleus has yet been conclusively shown to lie at the centre of an elliptical galaxy. To investigate the luminosity profile of the underlying galaxy circular aperture photometry was used to determine the fluxes in a number of concentric annuli centred on the emission centroid. Both elliptical and disk models of the brightness distribution have been fitted to the IR and optical data of the underlying galaxy. For the data presented the seeing was determined to be 3 arcsec FWHM from the analysis of a standard star. Rejecting the data with an effective radius $r<3$ arcsec, we found a de Vaucouleurs (DV) law is a better representation of the brightness distribution than the exponential disk models (fig.1.). In a more refined method to determine the true profile of the underlying galaxy we assumed the central flux is dominated by a point-like non-thermal nucleus. The point-spread function, PSF, as given by the standard star, was scaled to the nuclear peak and then subtracted directly to leave a residual profile to which we fitted DV and exponential laws. The height of the PSF was reduced until a best fit was acheived. To justify a more rigorous reduction we require images taken in improved seeing conditions. Radial colour gradients give mean colours of $B-V=0.40, J-H=0.61, H-K=0.26$ (all errors \pm 0.10 ) between 
radii of 4 and 20 arcsecs. We emphasis here, that as the brightness of the galaxy fades smoothly into the sky background, small errors in the sky level dramatically influences the appearance of both the luminosity profiles and the colour gradients at large radii. The IR colours of the outer regions of the underlying galaxy agree well with the canonical colours for elliptical and SO galaxies (Frogel et al. 1978). The optical colours are too blue for early type galaxies but are consistent with recent star formation fuelled by the accreted intracluster gas. We conclude that the azimuthal averaged brightness profiles and colours are more consistent with the underlying galaxy of NGC1275 being an elliptical.

The radius outside which the galactic emission is uncontaminated by the Seyfert nucleus is determined by comparison of the nuclear radial profile with the PSF, and by observing the dramatic increase in the radial colours at $\mathrm{r}<2$ arcsec. Assuming that the underlying galaxy is a normal elliptical and that ellipticals generally follow a DV law into the central arcsecs we have, as a first attempt to determine the non-thermal flux, extrapolated the model fit into the nucleus and subtracted off its contribution (ignoring the effects of seeing on the the form of the DV law in the nucleus). We show in fig. 2 the dominance of the non-thermal nucleus and the underlying stellar population in the 4 arcsec and 30 arcsec apertures respectively.

Finally we consider the infamous 'hotspot'. This has been explained as a foreground star, the nuclear remnant of a spiral galaxy, or a region of enhanced star formation fuelled by accreted gas (Metik \& Pronik, 1983). The B,V,J,H,K fluxes of this 'hotspot' are consistent with a foreground F8-G2 type star. Further spectroscopic studies are underway to confirm this conclusion.

\section{References}

Aaronson, M. 1977. PhD Thesis, Harvard University.

Frogel, J.A., Persson, S.E., Aaronson, M., \& Matthews, K. 1978. Astrophys. J., 220, 75.

Metik, L.P. \& Pronik, I.I. 1984. Astrofizika, 21, 233.

Minkowski, R. 1957. Radio Astronomy, I.A.U. Symp. No. 4, ed. H.C. van de Hulst (C.U.P.), p.107. 


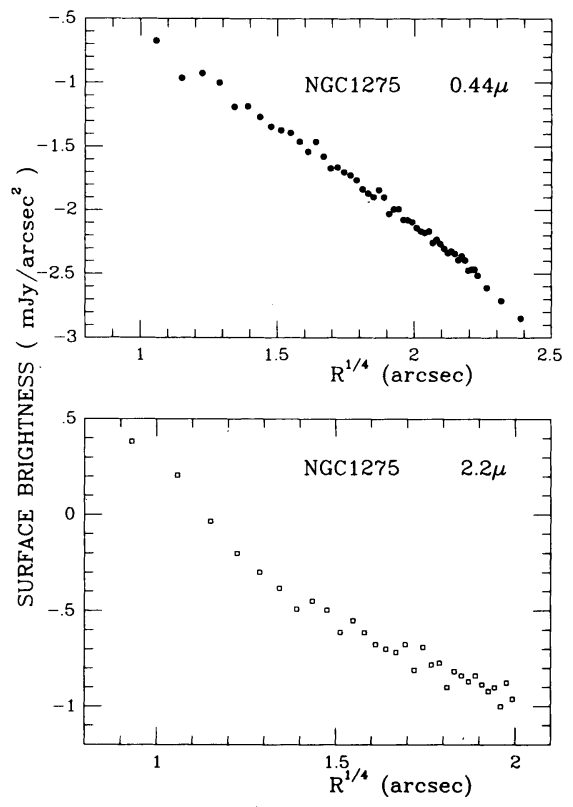

fig.1. Radial surface brightness distribution of NGC1275 at 0.44 and 2.2 microns.

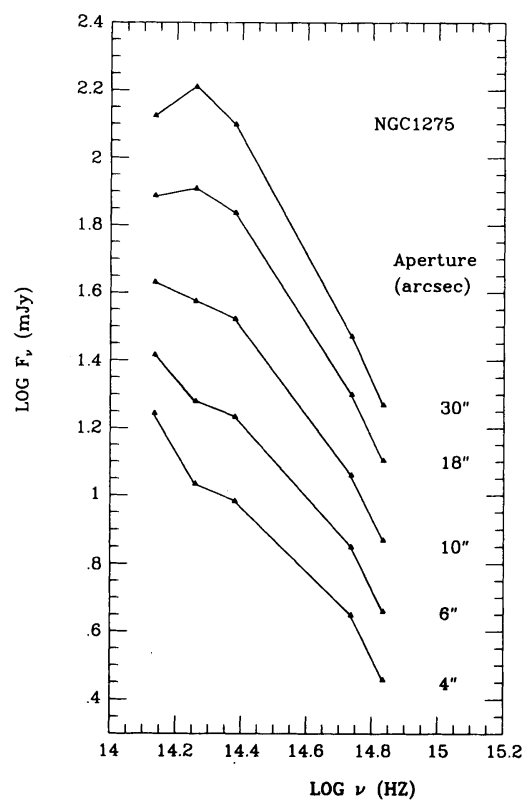

fig.2. Continuum energy distribution of NGC1275 in various apertures. 